Artículo científico

(Original paper)

\title{
PATRÓN TEMPORAL DE VOCALIZACIONES Y TERRITORIOS DE DESCANSO DEL TECOLOTE MOTEADO MEXICANO (STRIX OCCIDENTALIS LUCIDA NELSON 1903; STRIGIFORMES: STRIGIDAE) EN EL CENTRO-NORTE DE LA SIERRA MADRE OCCIDENTAL, MÉXICO
}

\author{
TEMPORAL PATTERN OF VOCALIZATIONS AND ROOSTING TERRITORIES OF \\ MEXICAN SPOTTED OWL (STRIX OCCIDENTALIS LUCIDA NELSON 1903; \\ STRIGIFORMES: STRIGIDAE) IN CENTRAL-NORTHERN OF THE SIERRA MADRE \\ OCCIDENTAL, MEXICO
}

\begin{abstract}
JAVIER RAFAEL-VALDEZ ${ }^{1}$, LUIS ANTONIO TARANGO-ARÁMBULA ${ }^{1}{ }^{*}$, JUAN FELIPE MARTÍNEZMONTOYA ${ }^{1}$, ARMANDO EQUIHUA-MARTÍNEZ ${ }^{2}$, OCTAVIO CESAR ROSAS-ROSAS ${ }^{1}$, GENARO OLMOSOROPEZA ${ }^{1}$

${ }^{1}$ Posgrado de Innovación en Manejo de Recursos Naturales, Colegio de Postgraduados, Campus San Luis Potosí. Iturbide No. 73, Salinas de Hidalgo, San Luis Potosí, CP. 78620, México.<rafael.javier@colpos.mx>; <ltarango@colpos.mx>; <fmontoya@colpos.mx>; <octaviocrr@colpos.mx>; <olmosg@colpo.mx>

${ }^{2}$ Posgrado en Entomología y Acarología, Colegio de Postgraduados Campus Montecillo. Carretera México-Texcoco km. 36.5. Colonia Montecillo, Texcoco, CP. 56230, México.<equihuaa@colpos.mx>

*Autor de correspondencia: <ltarango@colpos.mx>
\end{abstract}

Recibido: 24/05/2018; aceptado: 19/09/2019; publicado en línea: 26/09/2019

Editor responsable: Ricardo Rodríguez Estrella

Rafael-Valdez, J., Tarango-Arámbula, L. A., Martínez-Montoya, J. F., Equihua-Martínez, A., RosasRosas, C. O., Olmos-Oropeza, G. (2019) Patrón temporal de vocalizaciones y territorios de descanso del tecolote moteado mexicano (Strix occidentalis lucida Nelson 1903; Strigiformes: Strigidae) en el centro-norte de la Sierra Madre Occidental, México. Acta Zoológica Mexicana (nueva serie), 35, 1-15. https://doi.org/10.21829/azm.2019.3501221

RESUMEN. El tecolote moteado mexicano (TMM; Strix occidentalis lucida), para mantener comunicación con su pareja y tecolotes vecinos, emite vocalizaciones, las cuales varían con el sexo, la estación del año y condiciones climáticas. El TMM, para protegerse de depredadores y condiciones climáticas adversas, selecciona sitios específicos y establece territorios de descanso. Strix occidentalis lucida es una subespecie amenazada debido a la pérdida y fragmentación de sus hábitats. En México, los estudios sobre esta subespecie se refieren en su mayoría a la caracterización de su hábitat y composición de su dieta. Por ello, los objetivos de esta investigación fueron: a) monitorear la emisión de vocalizaciones, b) determinar el tamaño de los territorios de descanso diurno y c) identificar y describir las cuevas seleccionadas por Strix occidentalis lucida para perchar. Para ello, nueve parejas de TMM de tres regiones de la Sierra Madre Occidental se estudiaron de octubre de 2016 a octubre de 2017. El TMM emitió vocalizaciones durante todo el año, su territorio de descanso diurno promedio fue de $0.81 \pm 0.77$ ha y utilizó cuevas para descansar con mayor frecuencia en invierno (38.9\%) y primavera (33.3\%). Los resultados de este estudio son de utilidad para entender el comportamiento diurno del TMM y diseñar planes de manejo y conservación del hábitat del tecolote moteado mexicano en México.

Palabras clave: Especie amenazada; uso de cueva; sitio de descanso; actividad diurna 
Rafael-Valdez et al.: Patrón de vocalizaciones y territorios de descanso del

tecolote moteado mexicano

Rafael-Valdez, J., Tarango-Arámbula, L. A., Martínez-Montoya, J. F., Equihua-Martínez, A., RosasRosas, C. O., Olmos-Oropeza, G. (2019) Temporal pattern of vocalizations and roosting territories of Mexican spotted owl (Strix Occidentalis Lucida Nelson 1903; Strigiformes: Strigidae) in CentralNorthern of the Sierra Madre Occidental, Mexico. Acta Zoológica Mexicana (nueva serie), 35, 115. https://doi.org/10.21829/azm.2019.3501221

\begin{abstract}
The vocalizations of the Mexican spotted owl (TMM, Strix occidentalis lucida) are essential to maintain an effective and long-distance communication with your partner and neighboring owls, these vary depending on the season and the weather conditions that occur. In addition, their resting territories provide protection from predators and adverse climates. Studying vocalizations, resting places and territories are essential to know the biology of this subspecies with conservation risk. Strix occidentalis lucida is a threatened subspecies due to the loss and fragmentation of its habitats. In Mexico, studies on this subspecies refer mostly to the characterization of their habitat and composition of their diet. Therefore, the objectives of this investigation were: a) to monitor the emission of songs, b) to determine the size of the territories of daytime rest and c) to identify and describe the caves selected by Strix occidentalis lucida to rest. The samplings were conducted seasonally from October 2016 to October 2017 and consisted of monitoring nine pairs of TMM in three regions of the Sierra Madre Occidental. The TMM sang and used caves to rest throughout the year. The average size of its daytime resting territory was $0.81 \pm 0.77 \mathrm{ha}$. This subspecies used the caves more frequently in winter (38.9\%) and spring (33.3\%). The results of this study are useful to understand the diurnal behavior of the TMM and to design management and conservation plans for the Mexican spotted owl habitat in Mexico.
\end{abstract}

Key words: Endangered species; use of cave; resting place; daytime activity

\title{
INTRODUCCIÓN
}

El tecolote moteado mexicano (TMM; Strix occidentalis lucida) se distribuye en el suroeste de Estados Unidos de América (EUA) y en México (AOU, 2017). En EUA se le encuentra en Utah, Colorado, Arizona, Nuevo México y Texas (USFWS, 2012). En México se distribuye en la Sierra Madre Oriental, la Sierra Madre Occidental y el Eje Neovolcánico Trasversal en Aguascalientes, Chihuahua, Coahuila, Colima, Durango, Guanajuato, Jalisco, Michoacán, Nuevo León, San Luis Potosí, Sonora y Zacatecas (USFWS, 2012). El TMM es un ave territorial y de actividad nocturna, por lo que es más común escucharla que observarla (Ganey et al., 2013), y para comunicarse con su pareja y tecolotes vecinos, hace uso de vocalizaciones agudas y graves (Forsman et al., 1984). Estas vocalizaciones son más esenciales en áreas de vegetación densa (USFWS, 2012). Strix occidentalis lucida emite 12 tipos de vocalizaciones (Forsman et al., 1983), las cuales realiza después de la puesta del sol y antes del amanecer, principalmente de marzo a noviembre y con un aumento gradual de marzo a mayo (Ganey, 1990). Sus territorios de anidación y descanso (superficie que utiliza durante el día) los establece principalmente en ambientes rocosos con presencia de cuevas, los cuales presentan estructuras vegetales y microclimas propicias para esta subespecie (Ganey, 2004; Willey \& Van Riper, 2007). Estos ambientes, también les ofrecen protección contra depredadores y condiciones climáticas adversas (Tarango et al., 1997; Young et al., 1998; Tarango et al., 2001; Márquez et al., 2002; Palma et al., 2014); sin embargo, algunos TMM, durante el invierno, para sortear condiciones climáticas adversas, realizan migraciones altitudinales hacia zonas más cálidas (Ganey \& Block, 2005). En México esta subespecie se asocia con pino (Pinus spp.), encino (Quercus spp.), madroño (Arbutus unedo), ciprés de Arizona (Cupressus arizonica) y bosques de ribera (Alnus spp.), los cuales comúnmente son de dosel cerrado $41.2 \pm 24.7 \%$ y una densidad alta ( $47.7 \pm 44.5$ individuos/ha) de árboles muertos (Tarango et al., 2001; USFWS, 2012; Palma et al., 2014). A pesar de que Strix occidentalis tiene una distribución amplia, actualmente se clasifica en la categoría de especie amenazada (IUCN, 2019). En México, las causas principales para enlistar a esta subespecie de tecolote moteado como amenazada, son la pérdida y fragmentación de sus hábitats por la explotación forestal e incendios forestales (USDI, 1993; 
NOM-059 SEMARNAT, 2010). En EUA se han realizado estudios referentes a composición de la dieta del TMM, caracterización de sus sitios de descanso, estructura genética y sobre áreas de actividad (Bowden, 2008; Ganey et al., 2005; USFWS, 2012). Por el contrario, en México, sólo se han estudiado las características de sus sitios de descanso y composición de dieta (Young et al., 1997; Tarango et al., 1998; Tarango et al., 2001; Márquez et al., 2002; Bravo-Vinaja et al., 2005; Palma et al., 2014) y sus vocalizaciones fueron reportadas únicamente por Tarango (1994) en Chihuahua. Para lograr la permanencia de las poblaciones del TMM y mantener sus hábitats en condiciones adecuadas para su desarrollo, se requiere conocer más sobre su comportamiento y áreas de actividad. Por ello, los objetivos de esta investigación fueron: a) monitorear la emisión de vocalizaciones por el TMM, b) determinar el tamaño de sus territorios de descanso diurno y c) identificar y describir las cuevas seleccionadas por Strix occidentalis lucida para perchar. Para ello, nueve parejas de TMM de tres regiones de la Sierra Madre Occidental se estudiaron estacionalmente de octubre de 2016 a octubre de 2017. Los resultados de este estudio son de utilidad para entender el comportamiento diurno de $S$. o. lucida. Además, ayudan a definir las características de sus territorios de descanso.

\section{MATERIALES Y MÉTODOS}

Esta investigación se realizó en el centro-norte de la Sierra Madre Occidental de México. El área de estudio incluyó a: 1) El Área Natural Protegida (ANP); Región Prioritaria para la Conservación de Sierra Fría, Aguascalientes, 2) La Reserva de la Biósfera La Michilía y áreas adyacentes (Municipio de Súchil) en el estado de Durango y, 3) Tlachichila, Zacatecas (Fig. 1). El ANP Sierra Fría se ubica entre las coordenadas Latitud Norte: $21^{\circ} 52^{\prime} 45^{\prime}$ ' a 23० 31' 17' y Longitud Oeste: $102^{\circ} 22^{\prime} 44^{\prime \prime}$ a $102^{\circ} 50^{\prime} 53^{\prime \prime}$ e incluye los municipios de San José de Gracia, Rincón de Romos, Pabellón de Arteaga, Jesús María y Calvillo (CONANP, 2015). La Reserva de la Biósfera La Michilía y áreas adyacentes se localizan entre los paralelos $23^{\circ} 07^{\prime}$ y $23^{\circ} 43^{\prime}$ de latitud norte; los meridianos $103^{\circ} 47^{\prime}$ y $104^{\circ} 22^{\prime}$ de longitud oeste; (Anónimo, 2010a; INEGI, 2010). Tlachichila, Zacatecas se localiza al norte del municipio de Nochistlán de Mejía, en las coordenadas Latitud Norte: $21^{\circ} 32^{\prime} 31^{\prime \prime}$ a $21^{\circ} 40^{\prime}$ 03' ' y Longitud Oeste: $102^{\circ} 43^{\prime} 02^{\prime \prime}$ a $102^{\circ} 52^{\prime} 29^{\prime \prime}$ (Anónimo, 2010b; INEGI, 2010). El área de estudio tiene un clima templado (Cw; García, 2004), los principales hábitats son bosque de pino, bosque de pino-encino, matorral y agricultura de temporal (Anónimo, 2010a, b; CONANP, 2015).

El trabajo de campo se realizó de octubre de 2016 a octubre de 2017 durante las estaciones del año [otoño (23 de septiembre-21 de diciembre), invierno ( 21 de diciembre-21 de marzo), primavera (21 de marzo-21 de junio) y verano (21 de junio-23 de septiembre)], y consistió en dar seguimiento a nueve parejas de $S$. o. lucida (Fig. 1). Tres de ellas se localizaron en el ANP Sierra Fría, Aguascalientes (Tarango et al., 2001), tres en la Reserva de la Biósfera La Michilía y áreas adyacentes, Durango (Garza-Herrera, 1999) y tres en Tlachichila, Zacatecas (Palma et al., 2014). Para monitorear la emisión de vocalizaciones y verificar la ubicación de los tecolotes moteados mexicanos se realizaron 72 recorridos diurnos de 07:00 a 19:00 h [dos vistas a cada pareja por estación del año (a la mitad y al final de cada estación)]. Estos recorridos se realizaron a lo largo de senderos dentro del bosque e imitando sus cantos con un dispositivo musical (Bocina Bluetooth, marca: Link bits, modelo: RFR 023, rango: 300 m; Forsman et al., 1983), las vocalizaciones, en formato digital, fueron obtenidas de bibliotecas especializadas en sonidos de aves (www.xeno-canto.org). El reproductor de audio (volumen: alto) se orientó hacia los cuatro puntos cardinales durante diez minutos (se utilizaron vocalizaciones de hembras, machos y polluelos), después de las vocalizaciones se asignaron cinco minutos para escuchar y registrar la respuesta del tecolote moteado mexicano (cantos en el caso de adultos y silbidos para polluelos; USFWS, 2012). La localización de los tecolotes en sus sitios de percha comúnmente se realiza escuchando sus cantos durante el día o a través de la búsqueda de sus egagrópilas (regurgitaciones de componentes de dieta no digeridos). Para este estudio, realizado en el centro-norte de México, en virtud de que no existían reportes sobre la emisión de vocalizaciones del TMM y considerando que éstas disminuyen durante el otoño e invierno (Forsman et al., 1984; Ganey, 1990) o a que no las realizan 
Rafael-Valdez et al.: Patrón de vocalizaciones y territorios de descanso del tecolote moteado mexicano

(Tarango, 1994), se utilizó un perro entrenado (Golden retriver) para alternativamente localizar a los TMM a través de la ubicación de sus egagrópilas. En los recorridos de campo, cuando se obtuvo respuesta del tecolote, se registró la fecha, la hora, se observó el estado del tiempo (Ll: Lluvioso; Nb: Nublado; Sl: soleado; Pn: Parcialmente nublado) y con la ayuda del perro se inspeccionaron las áreas de donde provenían las vocalizaciones. Cuando los TMM se localizaron, el sitio de descanso o percha fue registrado, los tecolotes fueron contados y las coordenadas de ubicación UTM de cada pareja en su sitio de descanso o percha obtenidas con un GPS (Garmin 60CSx).

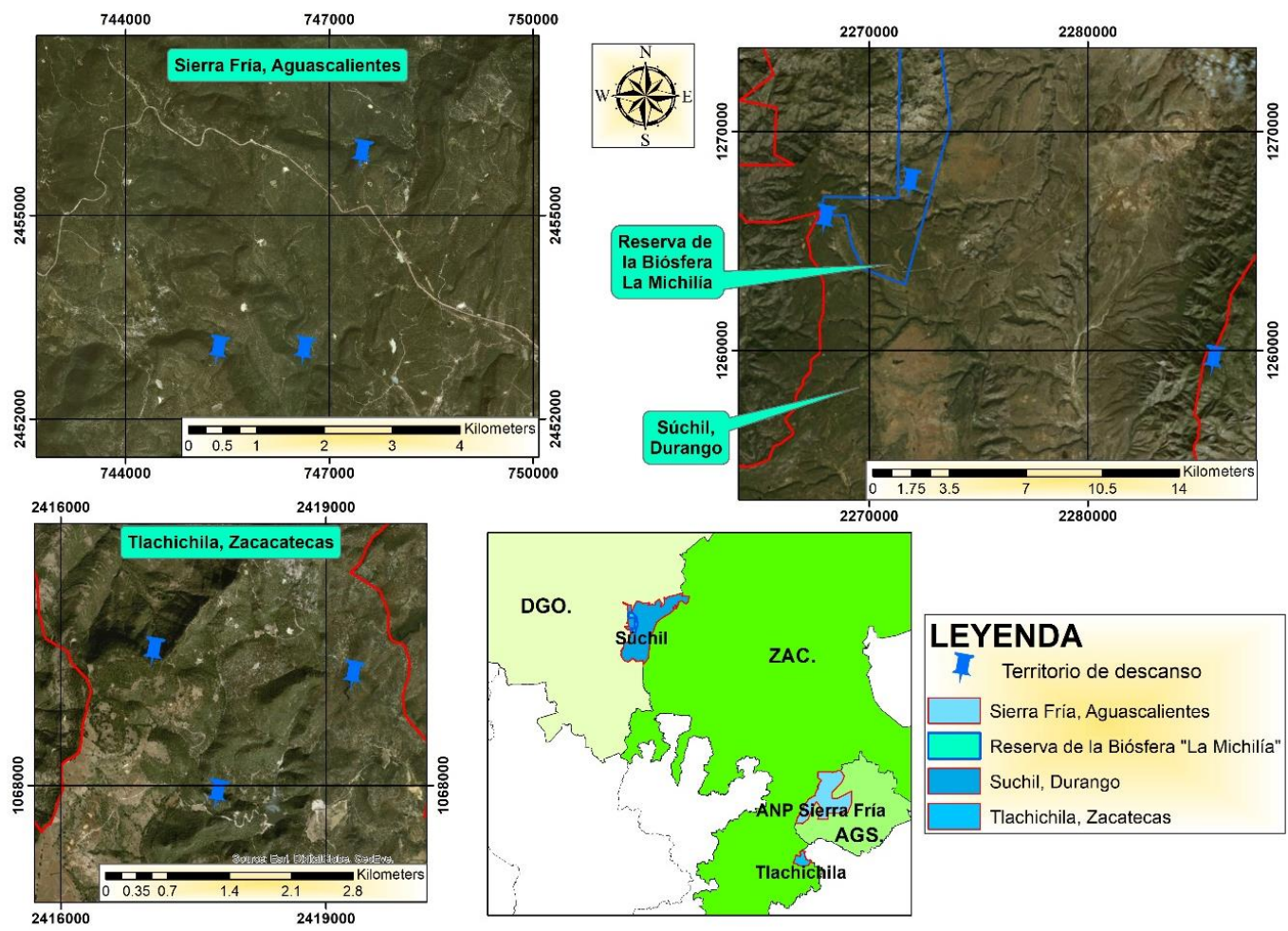

Figura 1. Área de estudio y territorios de descanso diurno del tecolote moteado mexicano en el centro-norte de la Sierra Madre Occidental, México.

Las vocalizaciones se ordenaron por estación (otoño, invierno, primavera y verano) del año y por época reproductiva (marzo-septiembre) y no reproductiva (octubre-febrero) del tecolote moteado mexicano (TMM). En virtud de que El TMM tiene una fidelidad muy marcada a sus territorios de descanso (Gutiérrez et al., 1995) y los defienden de otros individuos, ninguno de ellos fue marcado y se asumió que todos los individuos estudiados en cada sitio fueron los mismos durante el período de estudio.

Cabe señalar que en esta investigación no se determinó el ámbito hogareño del TMM, únicamente su territorio de descanso o percha diurno. Los polígonos de los territorios de descanso diurno de las parejas de TMM se determinaron a través del estimador Kernel (basados en una muestra aleatoria y una distribución no paramétrica) (Silverman, 1986; Worton, 1989) y con un 95\% de confiabilidad para representar áreas de uso concentrado (centros de actividad) en donde los TMM asignaron la mayor parte de su tiempo (Forsman et al., 2015). Para ello, la herramienta Kernel Density (Silverman, 1986; Worton, 1989; Ganey et al., 2005, Willey \& van Riper, 2007) del software ArcGis 10.5 (ESRI, 2016) y las coordenadas de cada sitio de descanso fueron utilizadas, las cuales se ordenaron por muestreo y estación. Por ejemplo, se realizaron dos muestreos por estación en cada uno de los sitios, cuando el muestreo se realizó a la mitad de la estación de otoño, éste se clasificó como Otoño 1 y cuando se realizó a finales como Otoño 2, y así sucesivamente para cada una de las estaciones. 
Asimismo, en cada sitio de descanso o percha del TMM el tipo de percha (árbol o cueva) fue determinado; cuando la percha fue un árbol, su especie fue identificada; cuando fue una cueva su a) orientación, b) largo (alto), c) ancho, d) profundidad de la cueva, e) altura a la entrada de la cueva, f) espesor del macizo rocoso sobre la cueva y g) altura total del macizo rocoso fueron medidas y registradas; también el tipo de vegetación donde se ubicaron los sitios de descanso fue registrado. Las mediciones se realizaron con un distanciómetro (Bushnell, Pro 1600 Slope edition Kansas, EE.UU.). Con la información de las variables tipo de percha y características de las cuevas, obtuvimos estadística descriptiva (medias y desviación estándar) con el uso del software Microsoft Excel® (Microsoft Office, 2016) y para identificar posibles diferencias en el uso de cuevas por el TMM por estación del año, se realizó un análisis de varianza $(\alpha=0.05)$ utilizando el software R-versión 3.2.0. (R core team, 2013).

\section{RESULTADOS}

En este estudio, durante octubre 2016 a octubre 2017, nueve parejas (18 tecolotes) de nueve territorios de descanso diurno del TMM de tres regiones de la Sierra Madre Occidental fueron estudiadas. Los nueve territorios de descanso y los 18 TMM se ubicaron en vegetación de pino-encino (Pinus spp. - Quercus spp.) (7 territorios; 14 tecolotes), encinos (Quercus spp.) (1 territorio; 2 tecolotes) y zona ribereña (Alnus spp.) (1 territorio; 2 tecolotes). Los tecolotes moteados mexicanos emitieron vocalizaciones durante las cuatro estaciones del año. Sin embargo, éstas fueron más frecuentes durante la época reproductiva (primavera y verano) con una respuesta de $94.4 \%$ en cada una de las estaciones comparadas con las registradas en la época no reproductiva (otoño e invierno) con un 55.6\% en otoño y un $66.7 \%$ en invierno. Cabe señalar que de las 72 visitas (18 por estación) a los territorios de descanso, en el $77.8 \%$ ( $\mathrm{n}=56$ ) de las ocasiones los TMM respondieron en condiciones de tiempo lluvioso, nublado, parcialmente nublado y soleado (Cuadro $1)$.

El territorio de descanso diurno del tecolote moteado mexicano varió de 0.03 a 1.85 ha $(0.81 \pm 0.77$ ha) (Cuadro 2). En el estado de Aguascalientes el territorio promedio fue de $1.09 \pm 0.84$ ha (Cuadro 1, Fig. 2), en Durango de $0.49 \pm 0.81$ ha (Cuadro 1, Fig. 3) y en Zacatecas de $0.84 \pm 0.88$ ha (Cuadro 1, Fig. 4).

El tecolote moteado mexicano, durante las cuatro estaciones del año, utilizó cuevas como sitios de descanso (Cuadro 3). En 18 de las 72 visitas a sus territorios se le encontró en ellas, con mayor frecuencia en el invierno (38.9\%) y primavera (33.3\%); sin embargo, el análisis de Kruskal-Wallis no identificó diferencias significativas $(p>0.999$; g.l. $=3)$ en el uso de cuevas por estación de muestreo.

Específicamente en tres territorios de Aguascalientes, uno de Durango y dos de Zacatecas (Figs. 5, 6), los TMM utilizaron diez cuevas (seis en Aguascalientes, una en Durango y tres en Zacatecas) para descansar. Las cuevas presentaron las siguientes características: a) orientación: norte $(\mathrm{n}=$ tres), noreste ( $\mathrm{n}$ $=$ cuatro), este $(\mathrm{n}=$ una), suroeste $(\mathrm{n}=$ una) y noroeste $(\mathrm{n}=$ una $)$; $\mathrm{b}) \operatorname{largo}($ alto $)=3.9 \pm 1.8 \mathrm{~m}, \mathrm{c})$ ancho $=$ $1.3 \pm 1.0 \mathrm{~m}, \mathrm{~d}$ ) profundidad $=3.1 \pm 1.5 \mathrm{~m}$, e) altura a la entrada de la cueva $=8.2 \pm 3.9 \mathrm{~m}, \mathrm{f}$ ) espesor del macizo rocoso sobre la cueva $=3.6 \pm 2.1 \mathrm{~m}, \mathrm{y} \mathrm{g}$ ) altura total del macizo rocoso $=15.0 \pm 4.5 \mathrm{~m}$.

\section{DISCUSIÓN}

Los tecolotes moteados mexicanos monitoreados durante este estudio vocalizaron durante las cuatro estaciones del año en todos los territorios, y contrario a lo reportado, vocalizaron bajo condiciones de lluvia, en días nublados, parcialmente nublados y soleados. Forsman et al. (1984) reportan que el TMM emite sus vocalizaciones entre marzo y noviembre, las cuales, para delimitar sus territorios, se incrementan durante el periodo de cortejo y anidación (marzo-mayo) y disminuyen durante junio-noviembre; asimismo, esta subespecie aumenta sus vocalizaciones en las noches con cielo despejado, sin viento y sin precipitación 
Rafael-Valdez et al.: Patrón de vocalizaciones y territorios de descanso del tecolote moteado mexicano

(Ganey, 1990). En Chihuahua, Tarango (1994) reportó vocalizaciones durante junio a septiembre de 1992, éstas se detuvieron de noviembre-1992 a febrero-1993 y continuaron el 17 de marzo de 1993. Es muy probable que por la ubicación geográfica del área de estudio y las condiciones climatológicas, el comportamiento de vocalización del tecolote moteado mexicano difiera con el de los Estados Unidos de América y el del norte de México (Tarango, 1994; USFWS, 2012). En Strix occidentalis las vocalizaciones son esenciales para identificar y estudiar sus territorios, y sitios de descanso y anidación, como la presente investigación.

Cuadro 1. Localización y respuesta a las vocalizaciones por el tecolote moteado mexicano en el centro-norte de la Sierra Madre Occidental, México.

\begin{tabular}{|c|c|c|c|c|c|c|c|c|c|c|c|}
\hline \multirow[b]{2}{*}{ Región } & \multirow{2}{*}{$\begin{array}{c}\text { Territorio } \\
\text { de } \\
\text { muestreo }\end{array}$} & \multicolumn{5}{|c|}{ Otoño } & \multicolumn{5}{|c|}{ Invierno } \\
\hline & & Fecha & Hora & Vocalizó & ET & NB & Fecha & Hora & Vocalizó & ET & NB \\
\hline \multirow{6}{*}{ Sierra Fría, Ags. } & BLS-M1 & $07 / 10 / 16$ & $14: 40$ & No & $\mathrm{Ll}$ & 0 & $12 / 02 / 17$ & $12: 33$ & $\mathrm{Si}$ & Sl & 1 \\
\hline & CP-M1 & $07 / 10 / 16$ & $17: 20$ & No & Sl & 1 & $12 / 02 / 17$ & $09: 41$ & $\mathrm{Si}$ & Sl & 2 \\
\hline & RA-M1 & SD & SD & SD & S1 & $\mathrm{SD}$ & $12 / 02 / 17$ & $16: 41$ & $\mathrm{Si}$ & S1 & 1 \\
\hline & BLS-M2 & $26 / 11 / 16$ & 09:30 & $\mathrm{Si}$ & $\mathrm{Nb}$ & 1 & $11 / 03 / 17$ & $12: 15$ & No & $\mathrm{Ll}$ & 1 \\
\hline & CP-M2 & $25 / 11 / 16$ & $13: 33$ & $\mathrm{Si}$ & Sl & 1 & $11 / 03 / 17$ & $16: 15$ & $\mathrm{Si}$ & $\mathrm{Nb}$ & 2 \\
\hline & RA-M2 & $26 / 11 / 16$ & $13: 24$ & No & Pn & 0 & $12 / 03 / 17$ & $14: 30$ & $\mathrm{Si}$ & S1 & 1 \\
\hline \multirow{6}{*}{ La Michilía, Dgo. } & EP-M1 & $21 / 10 / 16$ & $12: 53$ & No & Sl & 0 & $21 / 01 / 17$ & $12: 55$ & No & Sl & 0 \\
\hline & ET-M1 & $21 / 10 / 16$ & $17: 33$ & $\mathrm{Si}$ & S1 & 1 & $21 / 01 / 17$ & $18: 23$ & No & Sl & 2 \\
\hline & LR-M1 & $22 / 10 / 16$ & $13: 36$ & No & $\mathrm{S} 1$ & 0 & $22 / 01 / 17$ & $12: 55$ & $\mathrm{Si}$ & S1 & 2 \\
\hline & EP-M2 & $19 / 11 / 16$ & $15: 15$ & $\mathrm{Si}$ & $\mathrm{Pn}$ & 2 & $25 / 02 / 17$ & $13: 05$ & No & S1 & 0 \\
\hline & ET-M2 & $20 / 11 / 16$ & $09: 33$ & No & Pn & 0 & $25 / 02 / 17$ & $17: 57$ & $\mathrm{Si}$ & S1 & 1 \\
\hline & LR-M2 & $20 / 11 / 16$ & $14: 23$ & $\mathrm{Si}$ & $\mathrm{Pn}$ & 2 & $26 / 02 / 17$ & $12: 45$ & $\mathrm{Si}$ & S1 & 2 \\
\hline \multirow{7}{*}{ Tlachichila, Zac. } & BA-M1 & $09 / 10 / 16$ & $16: 23$ & $\mathrm{Si}$ & S1 & 1 & $14 / 01 / 17$ & $14: 49$ & $\mathrm{Si}$ & $\mathrm{Nb}$ & 2 \\
\hline & PP-M1 & $09 / 10 / 16$ & $11: 26$ & No & S1 & 2 & $15 / 01 / 17$ & $12: 37$ & No & $\mathrm{Nb}$ & 0 \\
\hline & RV-M1 & $09 / 10 / 16$ & $11: 46$ & $\mathrm{Si}$ & S1 & 1 & $14 / 01 / 17$ & $10: 57$ & $\mathrm{Si}$ & $\mathrm{Nb}$ & 1 \\
\hline & BA-M2 & $03 / 12 / 16$ & $15: 26$ & $\mathrm{Si}$ & $\mathrm{Ll}$ & 2 & $04 / 03 / 17$ & $16: 45$ & $\mathrm{Si}$ & $\mathrm{Nb}$ & 2 \\
\hline & PP-M2 & $04 / 12 / 16$ & $12: 35$ & $\mathrm{Si}$ & $\mathrm{Nb}$ & 2 & $04 / 03 / 17$ & $13: 45$ & No & $\mathrm{Nb}$ & 0 \\
\hline & RV-M2 & $03 / 12 / 16$ & $11: 56$ & $\mathrm{Si}$ & S1 & 1 & $04 / 03 / 17$ & $11: 05$ & $\mathrm{Si}$ & $\mathrm{Nb}$ & 1 \\
\hline & & \multicolumn{5}{|c|}{ Primavera } & \multicolumn{5}{|c|}{ Verano } \\
\hline \multirow{6}{*}{ Sierra Fría, Ags. } & BLS-M1 & SD & SD & SD & S1 & SD & $29 / 07 / 17$ & $10: 22$ & $\mathrm{Si}$ & Sl & 1 \\
\hline & CP-M1 & $29 / 04 / 17$ & $10: 30$ & $\mathrm{Si}$ & S1 & 2 & $29 / 07 / 17$ & $12: 43$ & $\mathrm{Si}$ & S1 & 2 \\
\hline & RA-M1 & $29 / 04 / 17$ & $14: 35$ & $\mathrm{Si}$ & Sl & 1 & $29 / 07 / 17$ & $14: 55$ & $\mathrm{Si}$ & S1 & 2 \\
\hline & BLS-M2 & $10 / 06 / 17$ & $08: 15$ & $\mathrm{Si}$ & $\mathrm{Ll}$ & 2 & $13 / 09 / 17$ & $11: 11$ & $\mathrm{Si}$ & S1 & 1 \\
\hline & CP-M2 & $10 / 06 / 17$ & $11: 57$ & $\mathrm{Si}$ & $\mathrm{Ll}$ & 2 & $13 / 09 / 17$ & $13: 56$ & $\mathrm{Si}$ & S1 & 2 \\
\hline & RA-M2 & $10 / 06 / 17$ & $14: 57$ & $\mathrm{Si}$ & $\mathrm{Ll}$ & 1 & $14 / 09 / 17$ & $15: 36$ & $\mathrm{Si}$ & Sl & 1 \\
\hline \multirow{6}{*}{ La Michilía, Dgo. } & EP-M1 & $13 / 05 / 17$ & $13: 23$ & $\mathrm{Si}$ & $\mathrm{Nb}$ & 1 & $05 / 08 / 17$ & $13: 13$ & $\mathrm{Si}$ & $\mathrm{Nb}$ & 2 \\
\hline & ET-M1 & $13 / 05 / 17$ & $17: 47$ & $\mathrm{Si}$ & $\mathrm{Nb}$ & 1 & $05 / 08 / 17$ & $18: 03$ & No & $\mathrm{Nb}$ & 0 \\
\hline & LR-M1 & $14 / 05 / 17$ & 13:07 & $\mathrm{Si}$ & S1 & 2 & $06 / 08 / 17$ & $13: 27$ & $\mathrm{Si}$ & S1 & 1 \\
\hline & EP-M2 & $17 / 06 / 17$ & $12: 33$ & $\mathrm{Si}$ & $\mathrm{S} 1$ & 2 & $21 / 09 / 17$ & $13: 42$ & $\mathrm{Si}$ & $\mathrm{Nb}$ & 1 \\
\hline & ET-M2 & $17 / 06 / 17$ & $18: 10$ & $\mathrm{Si}$ & $\mathrm{Sl}$ & 1 & $21 / 09 / 17$ & $10: 17$ & $\mathrm{Si}$ & Sl & 1 \\
\hline & LR-M2 & $18 / 06 / 17$ & $13: 17$ & $\mathrm{Si}$ & S1 & 2 & $22 / 09 / 17$ & $14: 20$ & $\mathrm{Si}$ & S1 & 1 \\
\hline \multirow{6}{*}{ Tlachichila, Zac. } & BA-M1 & $22 / 04 / 17$ & $16: 50$ & $\mathrm{Si}$ & $\mathrm{S} 1$ & 2 & $22 / 07 / 17$ & $17: 30$ & $\mathrm{Si}$ & $\mathrm{Nb}$ & 1 \\
\hline & PP-M1 & $23 / 04 / 17$ & $12: 17$ & $\mathrm{Si}$ & S1 & 2 & $23 / 07 / 17$ & $12: 09$ & $\mathrm{Si}$ & S1 & 1 \\
\hline & RV-M1 & $22 / 04 / 17$ & $11: 45$ & $\mathrm{Si}$ & $\mathrm{S} 1$ & 2 & $22 / 07 / 17$ & $15: 45$ & $\mathrm{Si}$ & $\mathrm{Nb}$ & 1 \\
\hline & BA-M2 & $03 / 06 / 17$ & $15: 23$ & $\mathrm{Si}$ & $\mathrm{Sl}$ & 1 & $16 / 09 / 17$ & $13: 41$ & $\mathrm{Si}$ & S1 & 1 \\
\hline & PP-M2 & $04 / 06 / 17$ & $13: 30$ & $\mathrm{Si}$ & Sl & 1 & $16 / 09 / 17$ & $16: 39$ & $\mathrm{Si}$ & Sl & 2 \\
\hline & RV-M2 & $04 / 06 / 17$ & $10: 20$ & $\mathrm{Si}$ & S1 & 2 & $15 / 09 / 17$ & $18: 07$ & $\mathrm{Si}$ & Sl & 1 \\
\hline
\end{tabular}

BLS: Barranca Laguna Seca; CP: Cueva Prieta; RA: Rancho Antrialgo; EP: El Purgatorio; ET: El Toribio; LR: Los Rebajes; BA: Barranca Angosta: PP: Peña Parda; RV: Rincón Verde; M1: Primer muestreo; M2: segundo muestreo; ET: Estado del tiempo; Ll: Lluvioso; Nb: Nublado; SI: soleado; Pn: Parcialmente nublado; NB: Número de búhos; SD: Sin datos (no muestreado por falta de acceso al área de estudio debido al traslape con la época de caza de venado cola blanca y guajolote silvestre).

En general la especie Strix occidentalis es muy territorial (más durante la época reproductiva), presenta una fidelidad a su territorio muy sobresaliente, en éste se le localiza año tras año y no se le considera 
una especie migratoria (Gutiérrez et al., 1995). Hamer et al. (2007) con la técnica de telemetría, determinaron que el espacio óptimo requerido (ámbito hogareño) por esta subespecie para llevar acabo sus actividades vitales varía con la estación; por ejemplo, durante el verano e invierno requirió en promedio $1,505 \pm 288$ y $2,920 \pm 868$ ha, respectivamente. En el presente estudio el territorio de descanso diurno varió entre 0.03 y 1.85 ha $(0.81 \pm 0.77 \mathrm{ha})$.

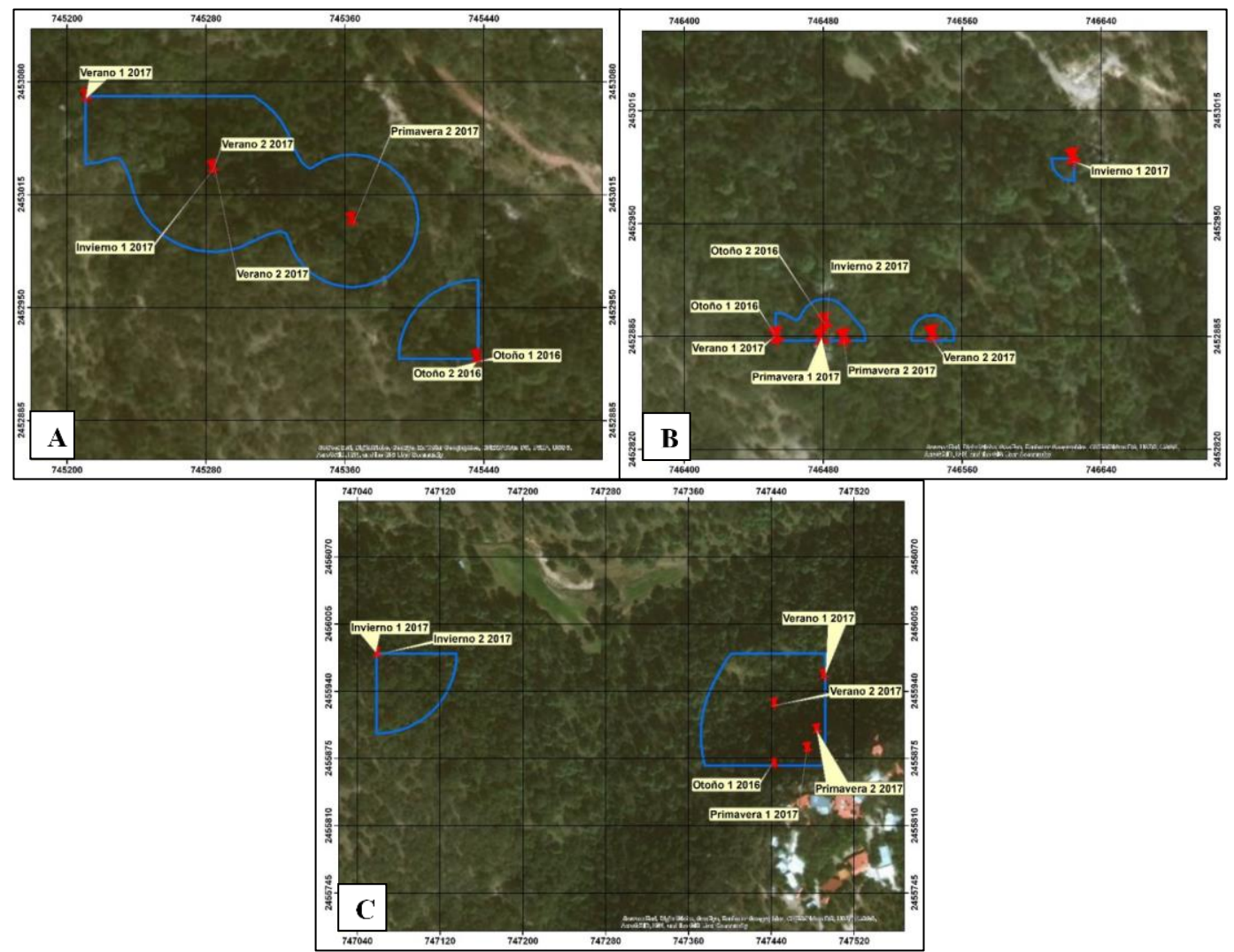

Figura 2. Territorios de descanso diurno (polígonos de líneas azules) del tecolote moteado mexicano (Strix occidentalis lucida) en Sierra Fría, Aguascalientes, México (A: Barranca Laguna Seca; B: Cueva Prieta; C: Rancho Antrialgo; puntos en rojo corresponden a los sitios en donde las parejas de TMM fueron localizadas por muestreo y estación).

Cuadro 2. Tamaño de los territorios de descanso diurno del tecolote moteado mexicano por estado y pareja en el centro-norte de la Sierra Madre Occidental, México.

\begin{tabular}{|c|c|c|c|c|}
\hline \multirow{2}{*}{ Región } & \multirow{2}{*}{ Estado } & \multicolumn{3}{|c|}{ Territorio de descanso diurno (ha) } \\
\hline & & Superficie & Pareja & Superficie \\
\hline \multirow{3}{*}{ Sierra Fría } & \multirow{3}{*}{ Aguascalientes } & \multirow{3}{*}{$1.09 \pm 0.84$} & *Barranca Laguna Seca & 1.46 \\
\hline & & & *Cueva Prieta & 0.13 \\
\hline & & & *Rancho Antrialgo & 1.69 \\
\hline \multirow{3}{*}{ La Michilía } & \multirow{3}{*}{ Durango } & \multirow{3}{*}{$0.49 \pm 0.81$} & El Purgatorio & 1.43 \\
\hline & & & *El Toribio & 0.03 \\
\hline & & & *Los Rebajes & 0.03 \\
\hline \multirow{3}{*}{ Tlachichila } & \multirow{3}{*}{ Zacatecas } & \multirow{3}{*}{$0.84 \pm 0.88$} & Barranca Angosta & 0.40 \\
\hline & & & Peña Parda & 0.28 \\
\hline & & & Rincón Verde & 1.85 \\
\hline
\end{tabular}

*Territorios de descanso sujetos a un plan de manejo y conservación. 
Rafael-Valdez et al.: Patrón de vocalizaciones y territorios de descanso del tecolote moteado mexicano

Cuadro 3. Tipo de percha del tecolote moteado mexicano por territorio de descanso en el centro-norte de la Sierra Madre Occidental, México.

\begin{tabular}{|c|c|c|c|c|c|}
\hline \multirow{2}{*}{ Región } & \multirow{2}{*}{$\begin{array}{c}\text { Territorio } \\
\text { de } \\
\text { descanso }\end{array}$} & \multicolumn{4}{|c|}{ Tipo de percha } \\
\hline & & Otoño & Invierno & Primavera & Verano \\
\hline \multirow{6}{*}{ Sierra Fría, Ags. } & BLS-M1 & $\begin{array}{c}\text { No } \\
\text { localizado }\end{array}$ & Cueva & SD & Quercus spp. \\
\hline & CP-M1 & Cueva & $\begin{array}{c}\text { Cueva y Quercus } \\
\text { spp. }\end{array}$ & Quercus spp. & Quercus spp. \\
\hline & RA-M1 & SD & Cueva & Quercus spp. & Quercus spp. \\
\hline & BLS-M2 & Cueva & Cueva & Quercus spp. & Quercus spp. \\
\hline & CP-M2 & Quercus spp. & Quercus spp. & Quercus spp. & Quercus spp. \\
\hline & RA-M2 & $\begin{array}{c}\text { No } \\
\text { localizado }\end{array}$ & Cueva & Arbutus unedo & Quercus spp. \\
\hline \multirow{6}{*}{$\begin{array}{l}\text { La Michilía, } \\
\text { Dgo. }\end{array}$} & EP-M1 & $\begin{array}{c}\text { No } \\
\text { localizado }\end{array}$ & No localizado & Pinus spp. & Pinus spp. \\
\hline & ET-M1 & Cueva & Cueva & Cueva & $\begin{array}{c}\text { No } \\
\text { localizado }\end{array}$ \\
\hline & LR-M1 & $\begin{array}{c}\text { No } \\
\text { localizado }\end{array}$ & Pinus spp. & Quercus spp. & Quercus spp. \\
\hline & EP-M2 & Quercus spp. & No localizado & Quercus spp. & Quercus spp. \\
\hline & ET-M2 & $\begin{array}{c}\text { No } \\
\text { localizado }\end{array}$ & Cueva & Cueva & Cueva \\
\hline & LR-M2 & Quercus spp. & Quercus spp. & $\begin{array}{c}\text { Pinus spp. y Quercus } \\
\text { spp. }\end{array}$ & Quercus spp. \\
\hline \multirow{5}{*}{ Tlachichila, Zac. } & $\begin{array}{l}\text { LBA- } \\
\text { M1 }\end{array}$ & Alnus spp & Alnus spp & Cueva & Alnus spp \\
\hline & PP-M1 & Quercus spp. & No localizado & Cueva & Quercus spp. \\
\hline & RV-M1 & Quercus spp. & Quercus spp. & Cueva & Quercus spp. \\
\hline & $\begin{array}{l}\text { LBA- } \\
\text { M2 }\end{array}$ & Alnus spp & Alnus spp & Cueva & Quercus spp. \\
\hline & $\begin{array}{l}\text { PP-M2 } \\
\text { RV-M2 }\end{array}$ & $\begin{array}{l}\text { Quercus spp. } \\
\text { Ouercus spn }\end{array}$ & No localizado & $\begin{array}{l}\text { Quercus spp. } \\
\text { Ouercus snp }\end{array}$ & Quercus spp. \\
\hline
\end{tabular}

BLS: Barranca Laguna Seca; CP: Cueva Prieta; RA: Rancho Antrialgo; EP: El Purgatorio; ET: El Toribio; LR: Los Rebajes; BA: Barranca Angosta: PP: Peña Parda; RV: Rincón Verde; M1: Primer muestreo; M2: segundo muestreo; NB: Número de búhos; SD: Sin datos (no muestreado por falta de acceso al área de estudio debido al traslape con la época de caza de venado cola blanca y guajolote silvestre).

De nueve territorios de descanso, cinco (tres en Aguascalientes y dos en Durango) se encuentran dentro de áreas naturales protegidas (ANP Sierra Fría y Reserva de la Biósfera La Michilía) que cuentan con un plan de manejo y conservación. Sin embargo, debido a los cambios frecuentes en el uso de suelo, a la explotación maderera clandestina e incendios forestales no controlados, los hábitats en los que el TMM se distribuye presentan diversos grados de disturbios y perturbación (Tarango et al., 1997; Márquez et al., 2002; Ganey et al., 2003; Silva-Piña et al., 2018); por esta razón, el tamaño de sus territorios de descanso diurno varío entre parejas de TMM y sitios. Es muy probable que el tamaño de los territorios determinados en esta investigación dependa del tamaño de muestra y del seguimiento temporal que se les dio a las parejas de tecolotes, el cual fue corto y puntual.

El plan de recuperación de Strix occidentalis lucida, indica que para conservar las zonas núcleo utilizadas por esta subespecie se deben establecer 243 ha (alrededor de los sitios de descanso), estas le brindarían territorios de anidación, descanso y forrajeo complementarios (USDI Federal Register, 1993). Al respecto Ganey et al. (2014) reportan que, en las montañas de Sacramento, Nuevo México, el tecolote moteado mexicano concentró sus actividades de anidación y de descanso dentro de las áreas recomendadas por el USDI y que otras áreas no habitadas por el TMM fueron recolonizadas. En Chihuahua, México, la 
Unidad de Manejo Forestal Número Cinco de San Juanito-Creel (UMAFOR; perteneciente a la Comisión Nacional Forestal) estableció parcelas de 30 ha alrededor de los sitios de descanso del tecolote moteado mexicano (Tarango, 1994), donde se prohíbe el aprovechamiento forestal. Sin embargo, esta superficie no es suficiente para asegurar la sobrevivencia de la especie.

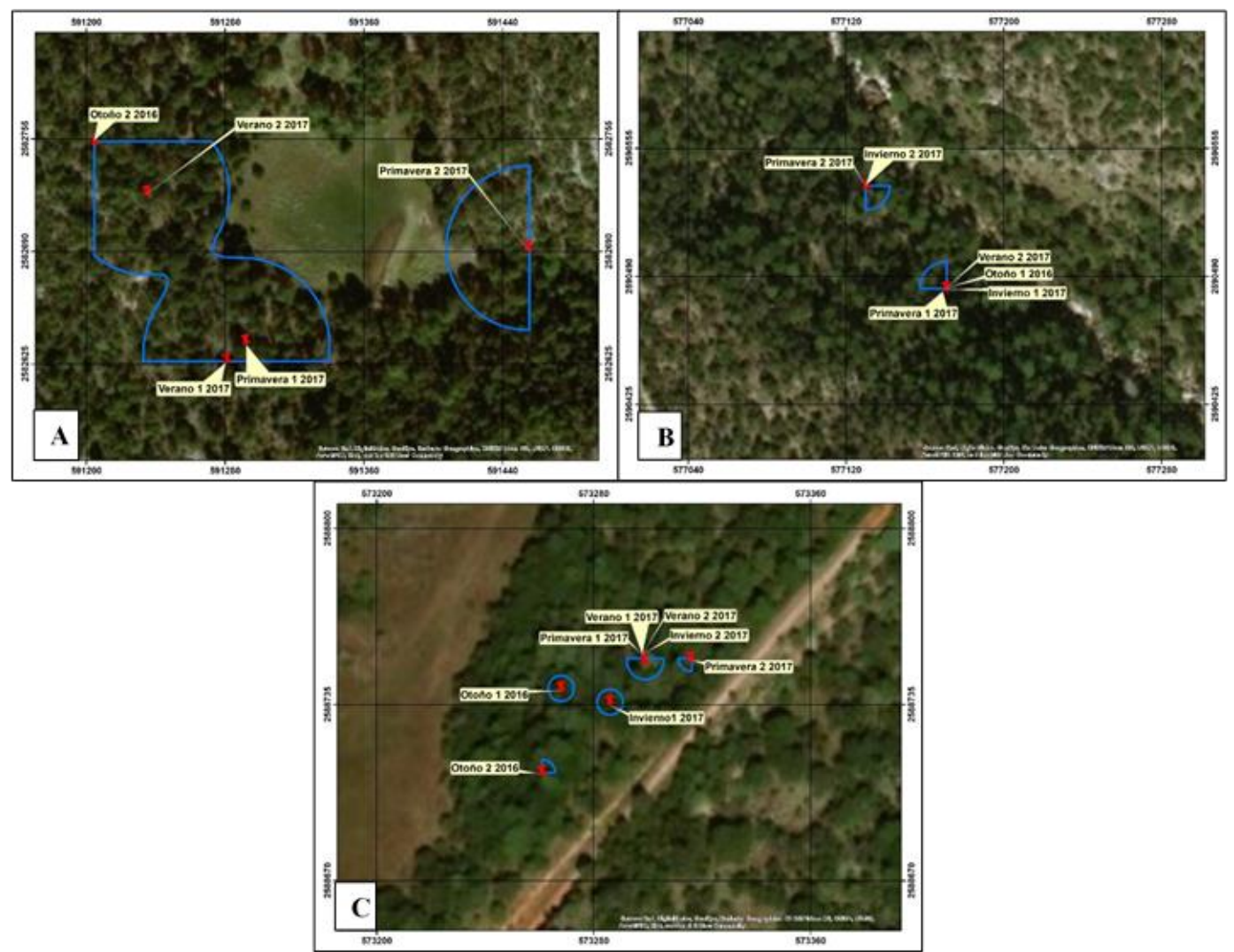

Figura 3. Territorios de descanso diurno (polígonos de líneas azules) del tecolote moteado mexicano (Strix occidentalis lucida) en la Reserva de la Biósfera La Michilia y áreas adyacentes, Durango, México (A: El Purgatorio;

B: El Toribio; C: Los Rebajes; puntos en rojo corresponden a los sitios en donde las parejas de TMM fueron localizadas por muestreo y estación).

En la presente investigación siete de los territorios de descanso se encontraron en bosque mixto de pino-encino (Pinus spp. - Quercus spp.). Al respecto, se ha determinado que los encinos (Quercus spp.) son un componente básico y un predictor de la selección de hábitat por esta subespecie (Forsman et al., 1984; Ganey et al., 2013); además, Seamans y Gutiérrez (1995) reportan que sus nidos se localizan comúnmente en bosques mixtos de coníferas donde el encino es una especie dominante o codominante del sotobosque.

Esta subespecie no construye nidos, utiliza estructuras naturales y prefabricadas por otras especies (Gutiérrez et al., 1995), anida en cuevas, en nidos construidos por otras aves, en las copas (plataformas de escombros) y cavidades de árboles. En este estudio al TMM no se le observó anidar en cuevas, pero si las utilizó para perchar. El TMM utilizó cuevas durante todo el año; sin embargo, las prefirieron durante el invierno posiblemente por las bajas temperaturas y en primavera por una escasa cobertura aérea de la vegetación de pino-encino (Pinus spp. - Quercus spp.) y de encino (Quercus spp.) requerida para regular su temperatura corporal (Barrows, 1981; Ganey et al., 1993). Ganey et al. (1988) reportaron que las temperaturas diurnas durante el verano en los territorios de descanso fueron menores a $25^{\circ} \mathrm{C}\left(1-6^{\circ} \mathrm{C}\right.$ más fríos que en las áreas más abiertas). Ganey et al. $(1999 ; 2003 ; 2013)$ reportan que, durante la temporada de reproducción, el TMM utiliza áreas con una cobertura de dosel $\geq 60 \%$ para forrajear y descansar; por el 
Rafael-Valdez et al.: Patrón de vocalizaciones y territorios de descanso del tecolote moteado mexicano

contrario, en la época no reproductiva puede utilizar áreas con una cobertura de 20-30\% (Ganey \& Block, 2005). Específicamente para las tres regiones estudiadas Silva-Piña et al. (2018) reportan una cobertura de dosel del $41.2 \pm 24.7 \%$. Sin embargo, algunos TMM realizan migraciones altitudinales durante el invierno a áreas con una estructura y composición de hábitats diferente a la de la época reproductiva (Ganey \& Block, 2005).

Las cuevas seleccionadas por el TMM presentaron orientación norte, noreste, este, suroeste y noroeste. Estos resultados son similares a los reportados para el estado de Chihuahua (Tarango et al., 1997), Aguascalientes (Tarango et al., 2001) y Zacatecas (Palma et al., 2014) quienes indican que el tecolote moteado mexicano prefiere laderas con exposición norte, noroeste y noreste que le provean microambientes más fríos. Los tecolotes del Parque Nacional del Gran Cañón anidan exclusivamente en acantilados y utilizan las rocas o cuevas como territorios de descanso (Bowden et al., 2015); aunque también ocupan hábitats fríos como bosques de coníferas y caducifolios con dosel cerrado y en laderas orientadas hacia el norte (Barrows, 1981; Ganey \& Balda, 1989); por el contrario, en Arizona y Nuevo México tienden a perchar en pendientes moderadas, orientadas al oeste, en las partes medias y altas de las laderas (Ganey et al., 2000). Esta subespecie selecciona hábitats con características que en conjunto le aseguren un microclima fresco (May et al., 2004). Ganey et al. (2000) mencionan que el bosque de coníferas mixto brinda las condiciones más estables y favorables para el tecolote moteado mexicano durante todo el año, mientras que los que habitan en los bosques de pino y encino se ven obligados a hacer ajustes estacionales en el uso de territorios de descanso.

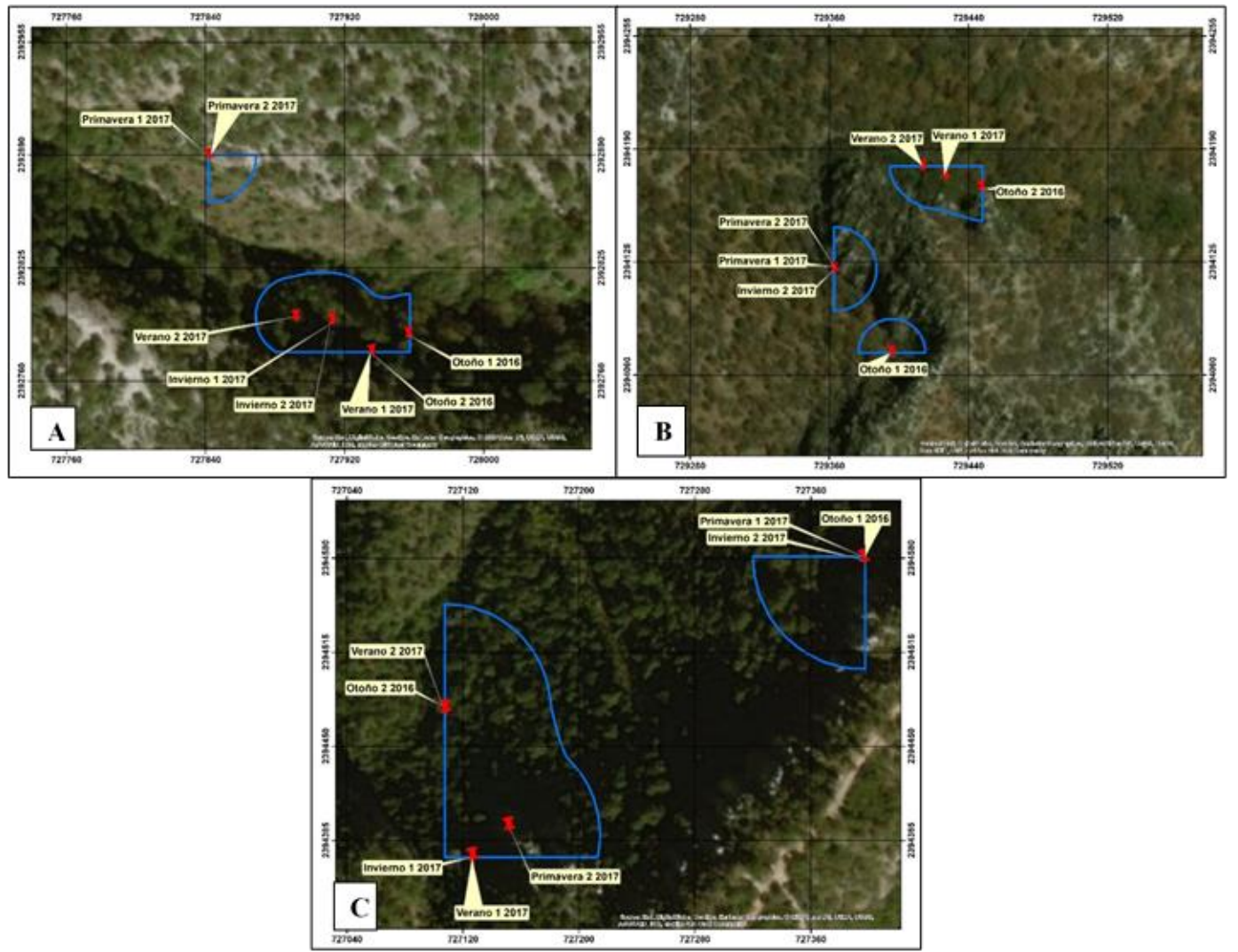

Figura 4. Territorios de descanso diurno (polígonos de líneas azules) del tecolote moteado mexicano (Strix occidentalis lucida) en Tlachichila, Zacatecas, México (A: Barranca Angosta; B: Peña Parda; C: Rincón Verde; puntos en rojo corresponden a los sitios en donde las parejas de TMM fueron localizadas por muestreo y estación). 


\section{Manejo y Conservación}

El TMM es una subespecie que habita ambientes templados diversos y que alcanza una vida de aproximadamente 15 años (USFWS, 2012); sin embargo, por asociarse a ecosistemas forestales de alta montaña es una subespecie susceptible al cambio climático (Villers \& Trejo, 2004), así como lo son otras especies que habitan los bosques templados. Asimismo, los cambios frecuentes en el uso del suelo, la explotación maderera e incendios forestales no controlados han provocado la pérdida y fragmentación de sus hábitats (USFWS, 2012). Por ello, mantener hábitats que permitan la sobrevivencia de esta especie es fundamental para su conservación a largo plazo. En este proceso, se deben tomar en cuenta los esquemas de desarrollo y ubicaciones topográficas de las comunidades vegetales en las que esta subespecie habita, así como conocer los patrones de movimientos estacionales del TMM.

El TMM, requiere un hábitat de tamaño adecuado (verano $1505 \pm 288$ ha e invierno $2910 \pm 868$ ha) que le pueda permitir el intercambio genético y crecimiento demográfico, asegurando la permanencia de su población y minimizando el riesgo de extinción (Hamer et al., 2007). Para ello, se requiere de una estrategia de conservación en la cual se protejan los hábitats potenciales de anidación, descanso y alimentación de las parejas reproductivas (USFWS, 2012). Esta estrategia, en general, debe enfocarse a crear y mantener hábitats del TMM de buena calidad (Ganey et al., 2005), y en aquellas áreas de distribución de esta subespecie en donde se practiquen actividades ganaderas, esta iniciativa debe considerar sistemas de pastoreo adecuados, y hacer un manejo y uso integral de los recursos naturales asociados al TMM.

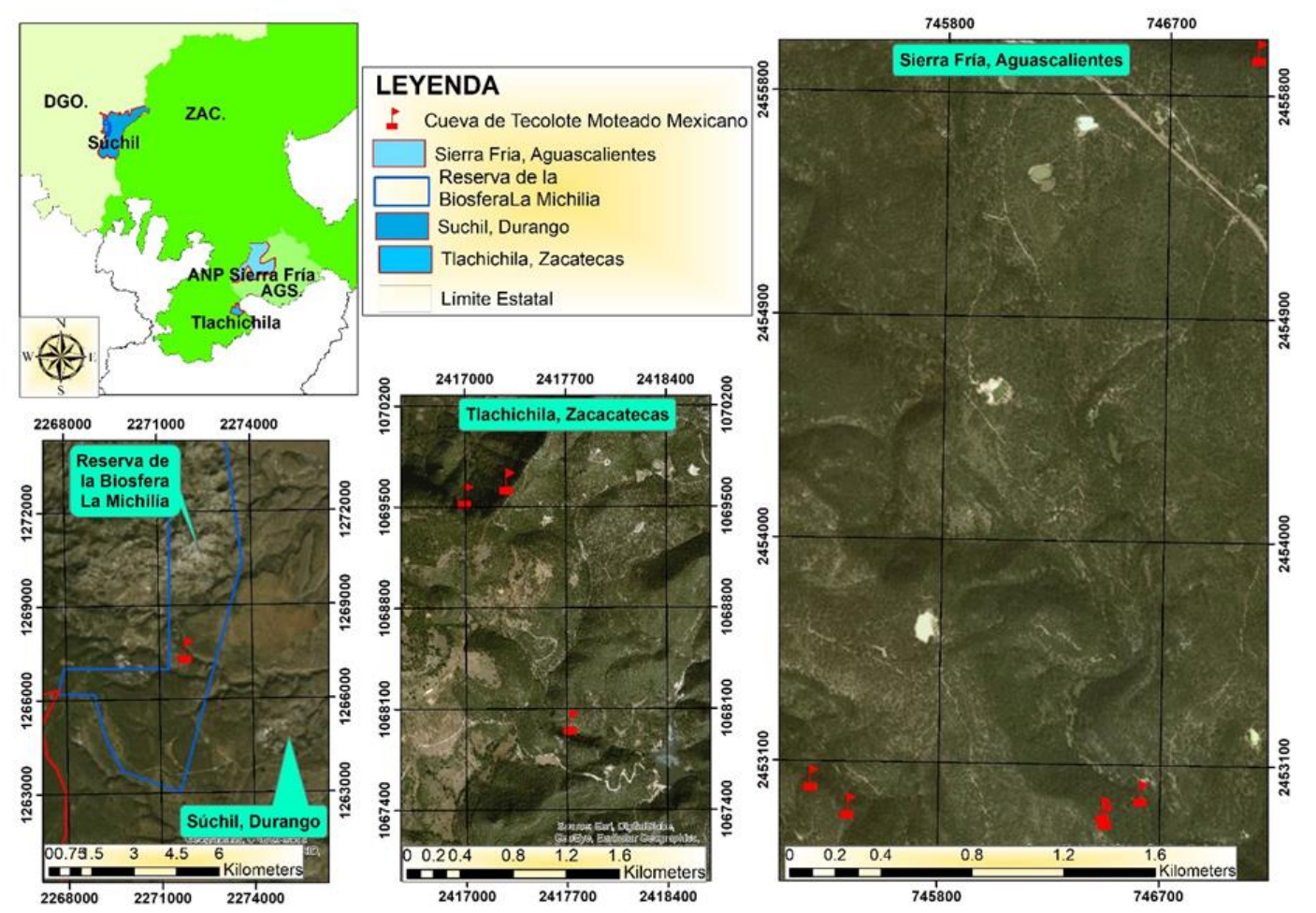

Figura 5. Localización de las cuevas utilizadas por el tecolote moteado mexicano (Strix occidentalis lucida) para descansar en el centro-norte de la Sierra Madre Occidental, México.

También es necesaria la coordinación eficiente entre el personal de la Comisión Nacional de Áreas Naturales Protegidas y de la Comisión Nacional Forestal para programar aprovechamientos forestales selectivos que mantengan condiciones óptimas para S. occidentalis lucida. Asimismo, es responsabilidad de las instituciones de investigación, de los gobiernos municipales, estatales y federales, continuar con acciones para preservar los hábitats y las poblaciones de esta subespecie en el territorio mexicano. 
Rafael-Valdez et al.: Patrón de vocalizaciones y territorios de descanso del tecolote moteado mexicano

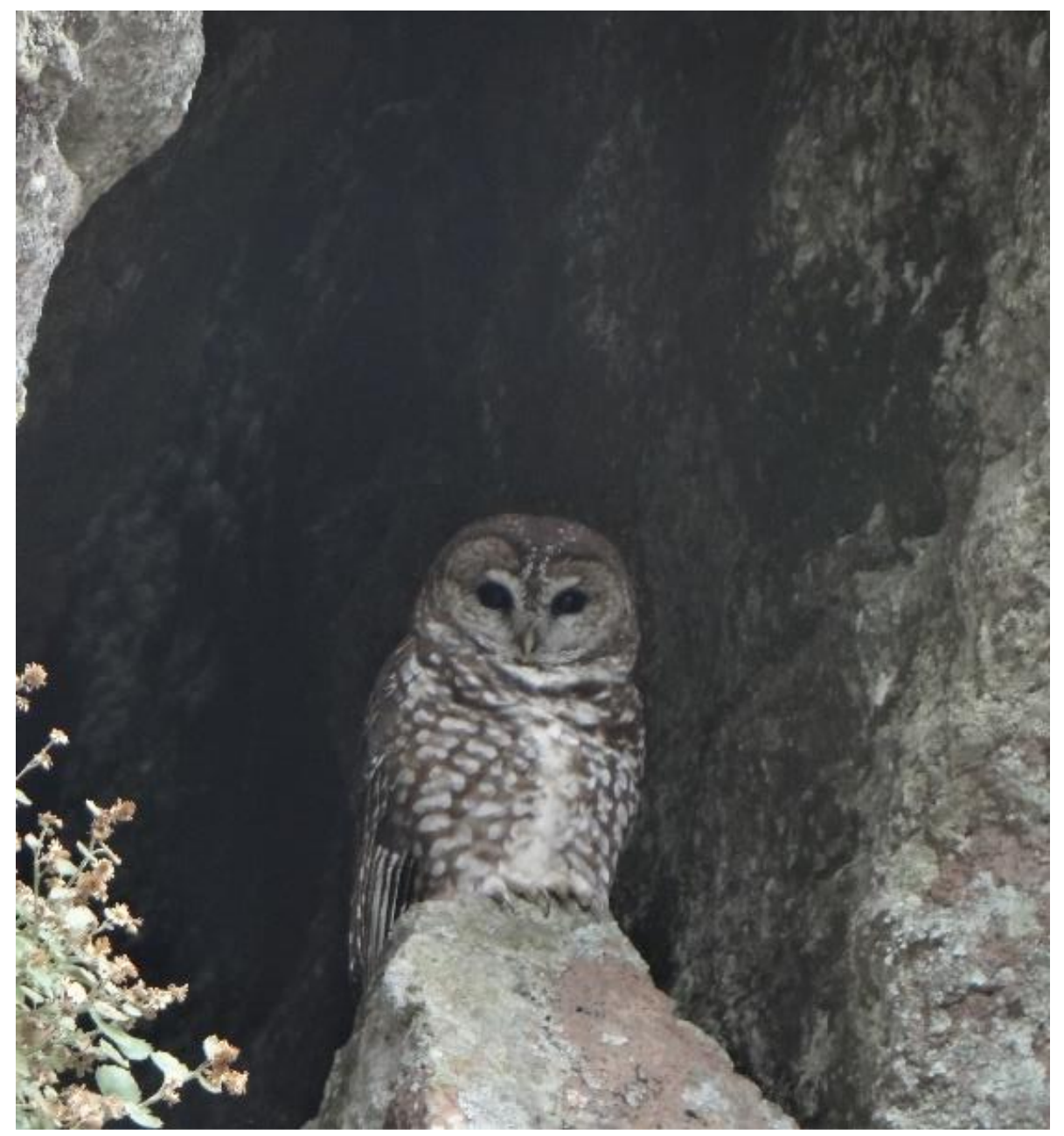

Figura 6. Cueva utilizada por Strix occidentalis lucida para descansar en Sierra Fría, Aguascalientes (Fotografía del primer autor).

\section{CONCLUSIONES}

El tecolote moteado mexicano (Strix occidentalis lucida), en el centro-norte de la Sierra Madre Occidental, México, vocalizó durante el día (77.8\%), en todas las estaciones del año e incrementó sus vocalizaciones; en la época reproductiva. Las vocalizaciones se registraron bajo condiciones meteorológicas de lluvia, nublado, parcialmente nublado y soleado. El territorio de descanso diurno de Strix occidentalis lucida fue de $0.81 \pm 0.77$ ha y utilizó las cuevas como percha, especialmente en invierno (38.9\%) y primavera (33.3\%).

Agradecimientos. Al Consejo Nacional de Ciencia y Tecnología (CONACYT) por la beca de postgrado otorgada al primer autor. Al Colegio de Postgraduados, Campus San Luis Potosí, por brindar las facilidades para el desarrollo del presente trabajo. Al Ing. Rodolfo Pineda Pérez, Director de la Reserva de La Biósfera La Michilía, Durango y al técnico Pedro Roldán Morales por su valioso apoyo en campo. Asimismo, al Sr. Alberto Guerrero Pérez, propietario del Rancho El Gauro, Julio Díaz Torre Macías y Jaime G. Díaz Torre Llamas, propietarios del Rancho Antrialgo en Sierra Fría, Aguascalientes por brindarnos su hospitalidad y acceso para la realización de la presente investigación. 


\section{LITERATURA CITADA}

Anónimo (2010a) Prontuario de información geográfica municipal de los Estados Unidos Mexicanos. Súchil, Durango. Disponible en: http://www3.inegi.org.mx/sistemas/mexicocifras/datosgeograficos/10/10033.pdf (Consultado el 10 de noviembre de 2017).

Anónimo (2010b) Prontuario de información geográfica municipal de los Estados Unidos Mexicanos. Nochistlán de Mejía, Zacatecas. Disponible http://www3.inegi.org.mx/sistemas/mexicocifras/datos-geograficos/32/32034.pdf (Consultado el 10 de noviembre de 2017).

AOU (American Ornithologists Union) (2017) Check-list of North American birds. 7ma edition. Washington, D.C. Disponible en: http://checklist.aou.org/taxa/ (Consultado el 22 de enero de 2018)

Barrows, C. W. (1981) Roost selection by spotted owls: an adaptation to heat stress. The Condor, 83, 302309.

Barrows, C., Barrows, K. (1978) Roost characteristics and behavioral thermoregulation in the spotted owl. Western birds, 9 (1), 1-8.

Bond, M. L., Lee, D. E., Siegel, R. B., Ward Jr, J. P. (2010) Habitat use and selection by California spotted owls in a Postfire Landscape. The Journal of Wildlife Management, 73 (7), 1116-1124.

Bowden, T. S. (2008) Mexican spotted owl reproduction, home range, and habitat associations in Grand Canyon National Park. Thesis. Montana State University, Bozeman, USA.

Bowden, T. S., Ferguson, J. M., Ward, R. V., Taper, M. L., Willey, D. W. (2015) Breeding season home range and habitat use of mexican spotted owls (Strix occidentalis lucida) below the South Rim of Grand Canyon National Park. The Wilson Journal of Ornithology, 127 (4), 678-689. https://doi.org/10.1676/15-004.1

Bravo-Vinaja, M. G., Tarango-Arámbula, L. A., Clemente-Sánchez, F., Mendoza-Martínez, G. D. (2005) Composición y variación de la dieta del tecolote moteado mexicano (Strix occidentalis lucida) en Valparaíso, Zacatecas, México. Agrociencia, 39 (5), 509-515.

CONANP (Comisión de Áreas Naturales Protegidas) (2015) Áreas Naturales Protegidas del estado de Aguascalientes. Disponible en: http://conacyt.gob.mx/cibiogem/index.php/anpl/aguascalientes (Consultado el 15 de noviembre de 2017).

ESRI (Environmental Systems Research Institute) (2016) ArcGIS Desktop: Release 10.5 Redlands.

Forsman, E. D. (1976) A preliminary investigation of the Spotted Owl in Oregon. M. S. Thesis, Oregon State Univ., Corvallis.

Forsman, E. D. (1983) Methods and materials, for locating and studying Spotted Owls. Gen. Tech. Rep. PNW-162. Portland, OR: US. Department of Agriculture, Forest Service, Pacific Northwest Forest and Range Experiment Station.

Forsman, E. D., Sovern, S. G., Taylor, M., Biswell, B. L. (2015) Home range and habitat selection by northern spotted owls on the eastern slope of the Cascade Mountains, Washington. Journal of Raptor Research, 49 (2), 109-129.

Forsman, E. D., Meslow, E. C., Wigth, H. M. (1984) Distribution and biology of the spotted owl in Oregon. Wildlife Monographs, 87, 1-64.

Ganey, J. L. (1990) Calling behavior of spotted owls in northern Arizona. The Condor, 92, 485-490.

Ganey, J. L. (2004) Thermal regimes of Mexican spotted owl nest stands. The Southwestern Naturalist, 49 (4), 478-486. https://doi.org/10.1894/0038-4909(2004)049<0478:TROMSO>2.0.CO;2

Ganey, J. L., Apprill, D. L., Rawlinson, T. A., Kyle, S. C., Jonnes, R. S., Ward, J. P. (2013) Nesting habitat of Mexican spotted owls in the Sacramento Mountains, New Mexico. Journal of Wildlife Management, 77 (7), 1426-1435. https://doi.org/10.1002/jwmg.599

Ganey, J. L., Balda, R. P. (1989) Distribution and habitat use of Mexican spotted owls in Arizona. The Condor, 91, 355-361. 
Rafael-Valdez et al.: Patrón de vocalizaciones y territorios de descanso del

tecolote moteado mexicano

Ganey, J. L., Block, W. M. (2005) Dietary overlap between sympatric Mexican spotted and great horned owls in Arizona. USDA Forest Service, General Technical Report, RMRSRP-57-WWW, Rocky Mountain Research Station, Fort Collins, Colorado, USA.

Ganey, J. L., Balda, R. P., King, R. M. (1993) Metabolic rate and evaporative water loss of Mexican spotted and great horned owls. Wilson Bulletin, 105, 645-656.

Ganey, J. L., Block, W. M., Ackers, S. H. (2003) Structural characteristics of forest stands within home ranges of Mexican spotted owls in Arizona and New Mexico. Western Journal of Applied Forestry, $18,189-198$.

Ganey, J. L., Block, W. M., Dwyer, J. K., Strohmeyer, B. E., Jenness, J. S. (1998) Dispersal movements and survival rates of juvenile Mexican spotted owls in northern Arizona. Wilson Bulletin, 110, 206217.

Ganey, J. L., Block, W. M., Jenness, J. S., Wilson, R. A. (1999) Mexican spotted owl home range and habitat use in pine-oak forest: implications for forest management. Forest Science, 45, 127-135.

Ganey, J. L., Block, W. M., King, R. M. (2000) Roost sites of radio-marked Mexican spotted owls in Arizona and New Mexico: sources of variability and descriptive characteristics. Journal Raptor Reserch, 34 (4), 270-278.

Ganey, J. L., Block, W. M., Ward Jr., J. P., Strohmeyer, B. E. (2005) Home range, habitat use, survival, and fecundity of Mexican spotted owls in the Sacramento Mountains, New Mexico. The Southwestern Naturalist, 50 (3), 323-333. https://doi.org/10.1894/0038-4909(2005)050[0323:HRHUSA]2.0.CO;2

Ganey, J. L., Johnson, J. A., Balda, R. P., Skaggs, R. W. (1988) Mexican spotted owl, pp. 145-150. En: Glinski, R. L., Pendleton, B. G., Moss, M. B., Le Franc, M. N., Millsap Jr. B. A., Hoffman, S. W. (Eds.). Proc. Southwest Raptor Management Symposium and Workshop Naturalist Wildlife Federal. Washington, D.C.

García, E. (2004) Modificaciones al sistema de clasificación climática de Köppen. Instituto de Geografía, Universidad Nacional Autónoma de México. Serie Libro N 6. México, D. F. 90 pp.

Garza-Herrera, A. (1999) Situación actual del búho manchado mexicano (Strix occidentalis lucida) y de los Strigiformes de la Reserva de la Biósfera La Michilía. Instituto de Ecología, A.C. Informe final SNIB-CONABIO proyecto No. H305. México D.F.

Gutiérrez, R. J., Franklin, A. B., La Haye, W. S. (1995) Spotted owl (Strix occidentalis). The birds of North America, number 179. The Academy of Natural Sciences Philadelphia and the American Ornithologists Union, Washington, DC, USA.

Hamer, H. T., Forsman, D. E., Glenn, M. E. (2007) Home Range attributes and habitat selection of barred owls and spotted owls in an area of sympatry. The Condor, 109 (4), 750-768. https://doi.org/10.1093/condor/109.4.750

INEGI (Instituto Nacional de Estadística y Geografía) (2010) Información nacional, por entidad federativa y municipios. Población, hogares y vivienda. Disponible en: http://www3.inegi.org.mx/sistemas/mexicocifras/ (Consultado el 10 de noviembre de 2017).

IUCN (International Union for Conservation of Nature and Natural Resources) (2019) The IUCN Red List of Threatened Species. Version 2019-1. Disponible en: https://www.iucnredlist.org

Márquez, O. M., Tarango, A. L. A., Mendoza, M. G. D. (2002) Caracterización del hábitat del tecolote moteado mexicano (Strix occidentalis lucida (X) Nelson, 1903) en Sierra Fría, Aguascalientes. Agrociencia, 36 (5), 541-546.

May, C. A., Petersburg, M. L., Gutiérrez, R. J. (2004) Mexican spotted owl nest- and roost-site habitat in northern Arizona. Journal Wildlife Management, 68, 1054-1064.

Microsoft Office (2016) Microsoft Excel software. Microsoft Excel for windows release 2016.

Palma, C. D. Y., Tarango, A. L. A., Ugalde, L. S., Alcántara, C. J. L., Ángeles, P. G., Ramírez, V. G., Martínez, M. J. F. (2014) Hábitat del tecolote moteado mexicano (Strix occidentalis lucida) en Tlachichila Zacatecas, México. Agroproductividad, 7 (4), 3-9.

R Core Team (2013) R: A language and environment for statistical computing. R Foundation for Statistical Computing, Vienna, Austria. Disponible en: http://www.Rproject.org/ (Consultado en mayo 2018). 
Seamans, M. E., Gutiérrez, R. J. (1995) Breeding Habitat of the Mexican spotted owl in the Tularosa Mountains, New Mexico. The Condor, 97 (4), 944-952.

SEMARNAT (Secretaría del Medio Ambiente y Recursos Naturales), Norma Oficial Mexicana-059 (2010) Protección ambiental-especies nativas de México de flora y fauna silvestres-categorías de riesgo y especificaciones para su inclusión, exclusión o cambio lista de especies en riesgo. Poder ejecutivo Federal. Secretaría del Medio Ambiente y Recursos Naturales. Diario Oficial de la Federación. 30 diciembre de 2010.

Silva-Piña, M. J. (2017) Hábitat y relación filogenética del tecolote moteado mexicano (Strix occidentalis lucida) en la Sierra Madre Occidental, México. Tesis de Maestría. San Luis Potosí, México, 109 pp.

Silva-Piña, M. J., Tarango-Arámbula, L. A., Clemente-Sánchez, F., Cortez-Romero, C., VelázquezMartínez, A., Rafael-Valdez, J., Ugalde-Lezama, S. (2018) Características del hábitat de sitios de descanso del búho manchado (Strix occidentalis lucida) en la Sierra Madre Occidental, México. Huitzil, Revista Mexicana de Ornitología, 19 (2), 141-156.

Silverman, B. W. (1986) Density estimation for statistics and data analysis. New York: Chapman and Hall, $176 \mathrm{pp}$.

Tarango, L. A. (1994) Mexican spotted owl distribution and habitat characteristics in southwestern Chihuahua, México. Thesis Master of science. New Mexico State University. Las Cruses, New Mexico. 85 pp.

Tarango, L. A., Valdez, R., Zwank, P. J., Cárdenas, M. (1997) Mexican spotted owl habitat characteristics in southwestern Chihuahua, Mexico. Southwestern Naturalist, 42 (2), 132-136.

Tarango, L. A., Valdez, R., Clemente, F., Mendoza, G. (2001) Roost-site characteristics of Mexican spotted owls in Sierra Fria, Aguascalientes, Mexico. Journal of Raptor Research, 35 (2), 165-168.

USDI, Fish and Wildlife Service (1993) Final rule to list the Mexican spotted owl as a threatened species. Federal Register, 58 (49), 14248-14271.

USFWS (United States Fish and Wildlife Service) (2012) Final Recovery Plan of the Mexican spotted owl (Strix occidentalis lucida), First Revision. U. S. Fish and Wildlife Service. Albuquerque, New Mexico. USA. 413 pp.

Villers, L., Trejo, I. (2004) Evaluación de la vulnerabilidad en los ecosistemas forestales. En: Martínez, J., Fernández, A. (Coords.). Cambio climático: una visión desde México. Instituto Nacional de Ecología, Secretaría de Medio Ambiente y Recursos Naturales, México. 239-254 pp.

Willey, D. W., Van Riper, C. (2007) Home range characteristics of Mexican spotted owls in the canyonlands of Utah. Journal of Raptor Research, 41, 10-15.

Worton, B. J. (1989) Kernel methods for estimating the utilization distribution in home-range studies. Ecology, 70, 164-168.

Young, K. E., Valdez, R., Zwank, P. J., Gould, W. R. (1998) Density and roost site characteristics of spotted owls in the Sierra Madre Occidental, Chihuahua, Mexico. The Condor, 100 (4), 732-736. https://doi.org/10.2307/1369756 